\title{
Ecological sounds affect breath duration more than artificial sounds
}

Mauro Murgia ${ }^{1,2}$, Ilaria Santoro ${ }^{1}$, Giorgia Tamburini ${ }^{1}$, Valter Prpic $^{1}$, Fabrizio Sors ${ }^{1}$, Alessandra Galmonte $^{3}$, Tiziano Agostini ${ }^{1}$

${ }^{1}$ Department of Life Sciences, University of Trieste, Trieste, Italy.

2 Department of Pedagogy, Psychology, Philosophy, University of Cagliari, Cagliari, Italy.

${ }^{3}$ Department of Neurological and Movement Sciences, University of Verona, Verona, Italy.

Accepted for publication in Psychological Research: $22^{\text {nd }}$ January 2015

Corresponding author: Mauro Murgia, PhD

Department of Life Sciences, University of Trieste

via Weiss, 21 - building $\mathrm{W}-34128$ Trieste (TS), Italy

E-mail: mauromurgianet@tiscali.it 


\begin{abstract}
Previous research has demonstrated that auditory rhythms affect both movement and physiological functions. We hypothesized that the ecological sounds of human breathing can affect breathing more than artificial sounds of breathing, varying in tones for inspiration and expiration. To address this question, we monitored the breath duration of participants exposed to three conditions: (a) ecological sounds of breathing, (b) artificial sounds of breathing having equal temporal features as the ecological sounds, (c) no sounds (control). We found that participants' breath duration variability was reduced in the ecological sound condition, more than in the artificial sound condition. We suggest that ecological sounds captured the timing of breathing better than artificial sounds, guiding as a consequence participants' breathing. We interpreted our results according to the Theory of Event Coding, providing further support to its validity, and suggesting its possible extension in the domain of physiological functions which are both consciously and unconsciously controlled.
\end{abstract}




\section{Introduction}

The use of auditory rhythmical cues for the modulation of movement and physiological parameters has been largely investigated in different domains. Most of previous studies used artificial cues, which provided the correct rhythm of the function to be modulated. However, these cues did not provide information related to individual's perceptual experience. On the contrary, only few studies used ecological cues providing rhythmical information related to individual's experience. In the present study we aimed to compare the effects of ecological and artificial rhythmical cues on breathing, in order to provide new empirical outcomes with relevant impact on both perception and action theories and clinical practice.

The effects of rhythmical cues on motor systems have been mainly supported by empirical evidences in the domain of neurophysiology and acoustic perception. For instance, neurophysiological studies demonstrated that listening to auditory rhythms recruits motor regions of the brain, such as supplementary motor area, midpremotor cortex, and cerebellum, even when no motor task is required (Chen et al. 2008; Stupacher et al. 2013). Such evidences suggest the existence of a particular connection between motor and acoustic areas of the brain, concerning the processing of rhythmical material.

The connection between motor and acoustic areas is further supported by the discovery of the mirror neurons (Rizzolatti et al. 1996; Rizzolatti and Craighero 2004). Those neurons activate in response to action observation and represent the neurological basis for imitation learning. Initially, the studies on mirror neurons focused on visual stimuli, whereas later the studies demonstrated the existence of audiovisual mirror neurons, which code actions independently of whether these actions are performed, heard, or seen (Kohler et al. 2002). The discovery of mirror 
neurons sensitive to auditory information provided new basis for the explanation of the mechanisms underlying the auditory modeling techniques.

The intervention techniques based on auditory modeling consist in the administration of auditory rhythmical cues which can guide the individuals' control of movements and/or physiological functions. Cognitive trainings based on auditory modeling have been developed and successfully tested in different domains. For instance, in neuropsychology, positive effects of auditory training were proved in motor rehabilitation of patients with Parkinson disease and strokes (Nombela et al. 2013; Thaut and Abiru 2010; Pelton et al. 2010); in sport psychology, performance improvements were found in hammer throw, swimming, rowing and many other sports, both in auditory and audiovisual conditions (Agostini et al. 2004; Murgia et al. 2014; Effenberg 2005; Schmitz et al. 2013); in music psychology, motionless listening improved motor sequence retention in novice musicians (Lahav et al. 2013); in clinical psychology and medicine, different device-guided breathing techniques facilitated patients' breathing control, inducing the synchronization of their own breathing rhythm with acoustically administered rhythms (Gavish 2010; Ekman et al. 2011, Mahtani et al. 2012; Ritz et al. 2013).

Most of the above mentioned intervention techniques used artificial sounds to shape motor/physiological outcomes. Those sounds are based on metronome or on sonified models of the actions to be reproduced. The sonified models usually convert the physical parameters of movement (force, velocity, etc.) in tones varying in pitch, loudness and other parameters of sound (Effenberg 2005). Therefore, those sounds provide participants with information related to the action to be produced, but they are quite different from the perceptual ecological experience of individuals. To the best of our knowledge, only a few studies used ecological sounds - that is the recording of sounds naturally produced by movements - to shape motor outcomes (Agostini et al. 
2004; Murgia et al. 2014). Like sonified models, those sounds provide participants with information related to the action to be produced, but they more accurately resemble the perceptual ecological experience of individuals.

As regards breathing, no study compared the effects of artificial and ecological sounds on the modulation of breathing. The comparison of the effects of these kinds of stimuli would be useful to better understand whether stimuli which are directly related to perceptual-motor experience (ecological sounds) may affect breathing, differently from stimuli which are only partially related to individual's experience (artificial sounds).

The matching between perceptual and motor experience is a core element in the Theory of Event Coding (Hommel et al. 2001; Zmigrod and Hommel 2009). Assuming that perception and action share a common representational system - as postulated by this theoretical framework ecological sounds should evoke a more accurate representation of movements than artificial sounds, due to the matching between auditory stimulation and previous motor experience (Murgia et al. 2012). This would increase the probability that individuals spontaneously follow the administered ecological rhythmical cues for pacing their movements. Instead, the artificial rhythmical cues would partially lack of matching with previous motor experience, reducing the probability for the artificial rhythms to be retrieved by the motor systems and, consequently, to be performed.

The same reasoning could be done for the physiological functions, such as breathing, which are both consciously and unconsciously controlled, and which are characterized by a strong rhythmical component. In this case, we can expect that listening to ecological sounds of breathing evokes a representation of breathing that matches with previous motor experience related to breathing. As a consequence, we expect that listening to ecological sounds of breathing would 
affect participants breathing more than artificial rhythmical sounds. In order to test whether the Theory of Event Coding can explain perceptual-motor phenomena in the domain of physiological functions, we compared the effects of ecological and artificial rhythmical sounds on breath duration, hypothesizing that ecological sounds reduce breath duration variability more than artificial rhythmical sounds.

\section{Materials and Methods}

\section{Participants}

Twenty volunteers (10 males, 10 females) participated in this experiment. Their age varied from 20 to 26 years $(M=22.12, S D=1.67)$. All participants indicated they had neither hearing limitations nor respiratory diseases. Participants also reported they had no experience with any breathing technique. Moreover, one healthy volunteer took part only in the stimuli generation phase. All participants were naïve as to the purpose of the experiment. The study was conducted according to the ethical guidelines for medical research involving human subjects. All Participants provided their written informed consent prior to inclusion in the study.

\section{Material and apparatus}

A microphone Stage Line ECM-925P was placed at a distance of 5 centimeters from the participants' nose and was used to record their own breathing sounds. The microphone was connected with a laptop computer Sony Vayo (Intel Core i7), and the Goldwave 5.58 software recorded the input signal. The same software was used to edit and to analyze the sounds. Finally an mp3 player Packard Bell Audiokey Premium Fm, connected with headphones Sennheiser HD515 (total harmonic distortion $<0.2 \%$ ) was used to administer the stimuli. Sennheiser HD515 
are circumaural (full size) headphones that completely surround the ear, providing a good level of isolation from environmental noise.

\section{Stimuli generation}

A database of ecological sounds was generated as follows. The sounds associated with breathing of a volunteer were recorded and temporally manipulated. The volunteer was required to arbitrary vary his breath duration, defined as the sum of the duration of sounds associated with inspiration and expiration. Amongst the recorded sounds, nine breath categories, whose duration ranged between 1.5 and 5.5 seconds equally spaced by 0.5 seconds $( \pm 0.02)$, were then selected.

Those sounds were the basis to create the database of ecological sound tracks of breaths. Those sound tracks had regular frequency and breath duration, and lasted ten minutes each. In order to create the ecological sound tracks, for each of the nine breath categories we manipulated the duration of the post-expiration pause - acoustically represented by a silent interval between two subsequent breaths - so that the breath frequencies per category ranged between 5 to 25 breaths per minute (when it was mathematically possible, depending on the breath duration). To manipulate the post-expiration pause, we calculated the multiplication of frequency per breath duration, we subtracted this overall duration from 60 seconds, and we divided the difference for the frequency. For instance, when the duration of the breath was equal to 3 seconds and we needed to create a sound corresponding to a breath frequency of 14 breaths per minute, we calculated the overall duration of the breaths in a minute (14 $\times 3=42$ seconds), then we subtracted $60-42=18$, finally we divided 18 per $14=1.28$ seconds. Therefore, we created a sound track having a post-expiration pause equal to 1.28 seconds. 
Similarly, we created a database of artificial sounds having identical duration and temporal frequency as the ecological sounds. Congruently with other studies using sonified tones to manipulate breathing duration (Meuret et al. 2001; Elliott and Izzo 2006; Gavish 2010; Ekman et al. 2011), rising tones indicated inspiration while falling tones indicated expiration. Artificial sounds' timbre consisted of a synthesized string instrument recorded with a professional keyboard synthesizer, Kurzweil PC3LE8. Sounds were single tones, recorded in the middle range octave, equally growing in pitch by 3 semitones for inspiration and decreasing by 3 semitones for expiration. The pitch of the artificial stimuli grow and decreased continuously as a glissando. In the end of the artificial sound session, we asked participants' opinion about the sonified breathing sounds provided in the experiment. All participants considered the artificial sounds as an accurate reproduction of the dynamic of breathing. Prototypical examples of artificial and ecological sounds used in the experiments are described in Figure 1.

Figure 1 about here

\section{Design and procedure}

A within-subject design was employed. The participants were exposed to three different conditions (ecological sound, artificial sound, and control), which were run over three consecutive session days, in counterbalanced order.

In order to accustom participants to the experimental setting and to make sure they had a sufficient rest before starting the experiment, participants were asked to lie down on a couch for six minutes before the beginning of each session. Each session consisted of two phases lasting six minutes each. The first phase was the same in the three sessions. Participants were asked to relax 
on the couch and breathe normally, while experimenters monitored the participants' breathing pattern. This phase served to collect the individual baseline levels of breathing duration and frequency, and to select from the database the sound closest to the individual breathing parameters at rest, for each participant in each session. The second phase varied according to the conditions.

In both ecological and artificial sound conditions, participants were asked to synchronize the beginning of their inspiration with the beginning of the inspiration sound, for each breath. Participants were not given any instruction regarding the breath duration (inspiration + expiration), so they could spontaneously determine the duration of their inspiration and expiration, reducing or augmenting the post-expiration pause. In this way, the beginning of the inspiration was directly imposed by the task, while the breath duration was not imposed and could be indirectly affected by the sound. The sound volume was set at a comfortable standard listening level that completely overcome participants' own breath sound and any other minor environmental noise. In the control condition participants were asked to breathe normally, like in the first phase, without following any instruction. By doing so, we could control for possible standardization of breathing due to the experimental setting. In every session, through the analysis of sounds, we measured the breath duration in both first and second phases. Data collection was double-blind.

\section{Results}

As preliminary analysis, we compared the recorded sounds of participants breath with the administered sounds, in both ecological and artificial sound conditions. This was necessary to control whether participants were really following the instructions (synchronization of the 
beginning of their inspiration with the beginning of the inspiration sound). This was a criteria for including participants in the data analyses. All participants met the criteria for being included in the analyses.

For statistical analyses, we considered the standard deviations of participants' breath duration, in each phase, Fig. 2a. We ran a 2 x 3 repeated measures ANOVA (Phase $\times$ Condition), and results evidenced a statistically significant interaction $\left[F(2)=5.235 ; p<.01 ; \eta^{2}=.22\right]$. In order to test our hypothesis, a set of paired samples t-test analyses were run to compare the differences between the first and the second phase, for each condition. We considered an alpha value adjusted with the Bonferroni method $(p=0.05 / 9$ comparisons $=0.0056)$. The results revealed that only in the ecological sound condition there was a significant reduction of breath duration variability between the first and the second phase $[t(19)=3.047 ; p<.005 ; d=.68]$. Moreover, comparing the breath duration variability of the first phases among the conditions, statistical analyses did not reveal any difference. As regards the second phases, we found that breath duration variability of the ecological sound condition was statistically lower than that of the control condition $[t(19)=3.348 ; p<.005 ; d=.75]$; while the breath duration variability of the artificial sound condition only appears lower than that of the control condition, but this comparison did not reach the adjusted alpha value $[t(19)=2.095 ; p=.025 ; d=.47]$.

In addition, we ran another set of analyses, adjusting the data for individual baseline levels of breath duration variability, by subtracting the standard deviation between the first and the second phase $\left(\mathrm{SD}_{1}-\mathrm{SD}_{2}\right)$, in order to obtain a "Reduction Of Variability" (ROV) index, Fig. 2b. A repeated measures ANOVA revealed the same significant effect as the interaction of the previous analysis $\left[F(2)=5.235 ; p<.01 ; \eta^{2}=.22\right]$, but new evidences emerged when comparing the pairs of conditions. As we did previously, we adjusted the alpha value with the Bonferroni method $(p=$ 
$0.05 / 3$ comparisons $=0.016)$. According to our hypothesis, we found that the ROV index of the ecological sound condition was higher than that of both control $[t(19)=2.860 ; p=.005 ; d=.76]$ and artificial sound $[t(19)=2.540 ; p<.01 ; d=.57]$ conditions.

Figure 2 about here

\section{Discussion}

The aim of the present study was to compare the effects of ecological and artificial rhythmical sounds on breath duration, hypothesizing that ecological sounds would affect breath duration more than artificial sounds. The results confirm our initial hypothesis. Indeed, we found that only in the ecological sound condition there was a significant reduction of breath duration variability between the first and the second phase, while it did not happen in both artificial sound and control conditions. Moreover, we found that the ROV index of the ecological sound condition was higher than that of the two other conditions. Therefore, we found empirical support for the hypothesis that ecological sounds affect breathing more than our artificial rhythmical sounds.

Our results are consistent with the theoretical framework proposed by Hommel and colleagues (Hommel et al. 2001). Indeed, the timing of breathing is better captured by ecological than by our artificial sounds. It suggests that the representation of breathing evoked by ecological sounds better overlaps motor representation of breathing, as predicted by the Theory of Event Coding. Thus, the present work provides further evidence in support of the functional architecture proposed by Hommel and colleagues, and suggests a possible extension of its validity in the domain of physiological functions. However, future experiments should further investigate this hypothesis, providing new empirical evidences supporting or disconfirming the validity of the 
Theory of Event Coding for other physiological functions which are both consciously and unconsciously controlled.

Another important aspect regards the nature of the artificial stimuli. We decided to manipulate pitch height for simulating the dynamic of breathing, thus maintaining continuity with previous research studies in this field (Gavish 2010; Ekman et al. 2011; Mahtani et al. 2012; Ritz et al. 2013). However, there may be other typologies of artificial stimuli, that modifies different sound parameters, which could have different effects on modulating the breath duration. Therefore, future studies should investigate the effects of different artificial sounds on breath duration.

Moreover, as regards the future research developments, it would be interesting to test the activation of the individuals' brain areas associated to the mirror-neuron system (Rizzolatti et al. 1996; Rizzolatti and Craighero 2004), during the listening to ecological and artificial sounds of breathing. According to the general principles of the audiovisual mirror neurons (Kohler et al. 2002), it should be reasonable to expect a higher involvement of these brain areas during the administration of ecological sounds, rather than of artificial sounds.

As concerns practical implications, the outcomes of the present study may have a relevant influence on the development of intervention techniques based on auditory modeling. Indeed, the efficacy of ecological sound trainings - compared to artificial sound trainings - should be tested in the domains of motor rehabilitation, sport performance, and cognitive treatment of psychological disorders. If results confirmed the major influence of ecological sounds over the artificial ones, the current intervention techniques could be improved by adopting ecological sounds.

Concluding, the strength of the present study is its focus on ecological aspects, compared to previous studies on breathing modulation. We were interested in testing the effects of different 
sounds on breath duration, and found that ecological sounds have a stronger effect, compared to our artificial sounds. Our results suggest that the representation of ecological sounds better overlapped the internal representation of breathing (given by previous motor experience), compared with the representation of our artificial sounds, which was only partially related to perceptual-motor experience.

\section{Acknowledgments}

We would like to thank Bernhard Hommel and two anonymous reviewers for their valuable suggestions. 


\section{References}

Agostini, T., Righi, G., Galmonte, A., \& Bruno, P. (2004). The relevance of auditory information in optimizing hammer throwers performance. In P.B. Pascolo (Ed.), Biomechanics and Sports (pp 67-74). Vienna: Springer.

Chen, J.L., Penhune, V.B., \& Zatorre, R.J. (2008). Listening to Musical Rhythms Recruits Motor Regions of the Brain. Cerebral Cortex, 18, 2844-2854.

Effenberg, A.O. (2005). Movement Sonification: Effects on Perception and Action. IEEE Multimedia, 12, 53-59.

Ekman, I., Kjellström, B., Falk, K., Norman, J., \& Swedberg, K. (2011). Impact of device-guided slow breathing on symptoms of chronic heart failure: a randomized controlled feasibility study. European Journal of Heart Failure, 13, 1000-1005.

Elliott, W. J., \& Izzo, Jr, J. L. (2006). Device-guided breathing to lower blood pressure: case report and clinical overview. Medscape General Medicine, 8(3), 23.

Gavish, B. (2010). Device-guided breathing in the home setting: Technology, performance and clinical outcomes. Biological Psychology, 84, 150-156.

Hommel, B., Müsseler, J., Aschersleben, G., \& Prinz, W. (2001). The Theory of Event Coding (TEC): a framework for perception and action planning. Behavioral and Brain Sciences, 24, 849-878.

Kohler, E., Keysers, C., Umiltà, M.A., Fogassi, L., Gallese, V., et al. (2002). Hearing Sounds, Understanding Actions: Action Representation in Mirror Neurons. Science 297, 846-848.

Lahav, A., Katz, T., Chess, R., \& Saltzman, E. (2013). Improved motor sequence retention by motionless listening. Psychological Research, 77, 310-319. 
Mahtani, K.R., Nunan, D., \& Heneghan, C.J. (2012). Device-guided breathing exercises in the control of human blood pressure: systematic review and meta-analysis. Journal of Hypertension, 30, 852-860.

Meuret, A. E., Wilhelm, F. H., \& Roth, W. T. (2001). Respiratory biofeedback-assisted therapy in panic disorder. Behavior Modification, 25(4), 584-605.

Murgia, M., Forzini, F., \& Agostini, T. (2014). Migliorare le prestazioni sportive. Superare il doping con la psicologia sperimentale applicata al movimento. Milan: FrancoAngeli.

Murgia, M., Hohmann, T., Galmonte, A., Raab, M., \& Agostini, T. (2012). Recognising one's own motor actions through sound: the role of temporal factors. Perception, 41, 976-987.

Nombela, C., Hughes, L.E., Owen, A.M., \& Grahn, J.A. (2013). Into the groove: can rhythm influence Parkinson's disease? Neuroscience and Biobehavioral Reviews, 37, 2564-2570.

Pelton, T.A., Johannsen, L., Chen, H., \& Wing, A.M. (2010). Hemiparetic stepping to the beat: asymmetric response to metronome phase shift during treadmill gait. Neurorehabilitation and Neural Repair, 24, 428-434.

Ritz, T., Meuret, A.E., Bhaskara, L., \& Petersen, S. (2013). Respiratory muscle tension as symptom generator in individuals with high anxiety sensitivity. Psychosomatic Medicine, 75, 187195.

Rizzolatti, G., \& Craighero, L. (2004). The mirror-neuron system. Annual Review of Neuroscience, $27,169-192$.

Rizzolatti, G., Fadiga, L., Gallese, V., \& Fogassi, L. (1996). Premotor cortex and the recognition of motor actions. Cognitive Brain Research, 3, 131-141. 
Schmitz, G., Mohammadi, B., Hammer, A., Heldmann, M., Samii, A., et al. (2013). Observation of sonified movements engages a basal ganglia frontocortical network. BMC Neuroscience, 14, 32.

Stupacher, J., Hove, M.J., Novembre, G., Schütz-Bosbach, S., \& Keller, P.E. (2013). Musical groove modulates motor cortex excitability: A TMS investigation. Brain and Cognition, 82, 127-136.

Thaut, M.H., \& Abiru, M. (2010). Rhythmic Auditory Stimulation in Rehabilitation of Movement Disorders: A Review Of current research. Music Perception, 27, 263-269.

Zmigrod, S., \& Hommel, B. (2009). Auditory event files: Integrating auditory perception and action planning. Attention, Perception \& Psychophysics, 71, 352-362. 


\section{Figure captions}

Fig. 1 Prototypical examples of the artificial (on the left) and ecological sounds (on the right) used in our study. The upper graphs describe the amplitude of the sounds (ordinate axis); the lower graphs describe their frequencies (ordinate axis). In both cases the abscissa axis represents the time.

Fig. 2 Breath duration variability in the three conditions. The first graph (a) shows the average standard deviations of breathing duration variability in both first and second phases. The second graph (b) shows the Reduction Of Variability (ROV) index - obtained by the difference between the standard deviations of phase 1 and those of phase 2 for each condition. Error bars indicate standard errors. 


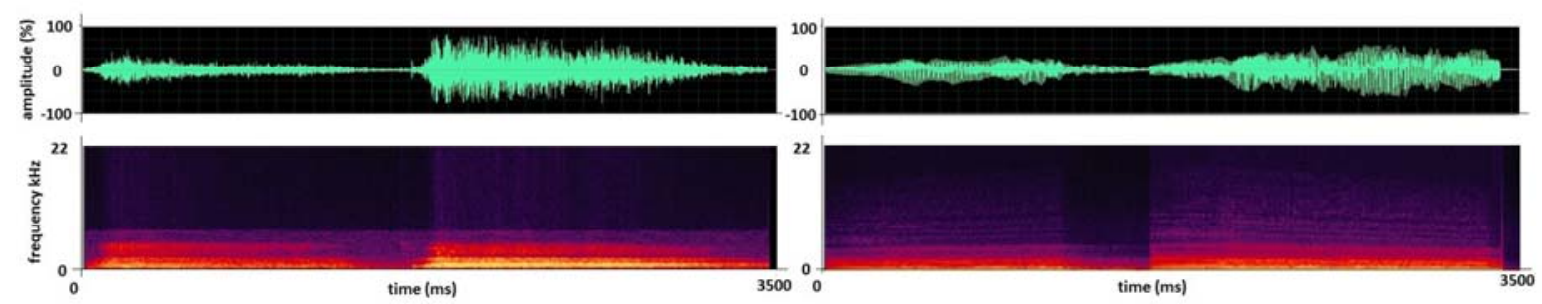

Figure 1

a

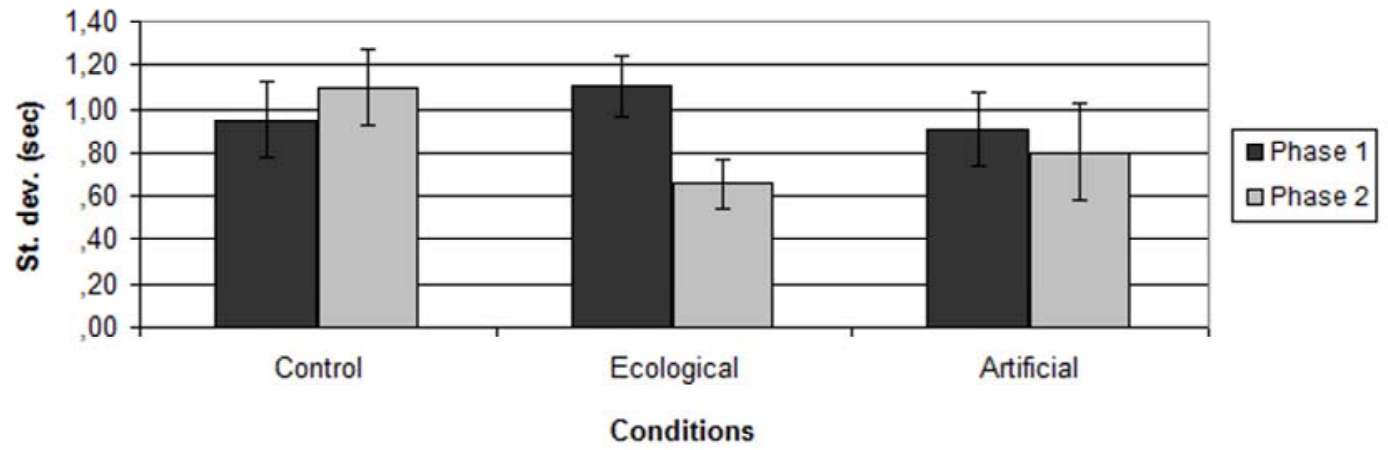

b

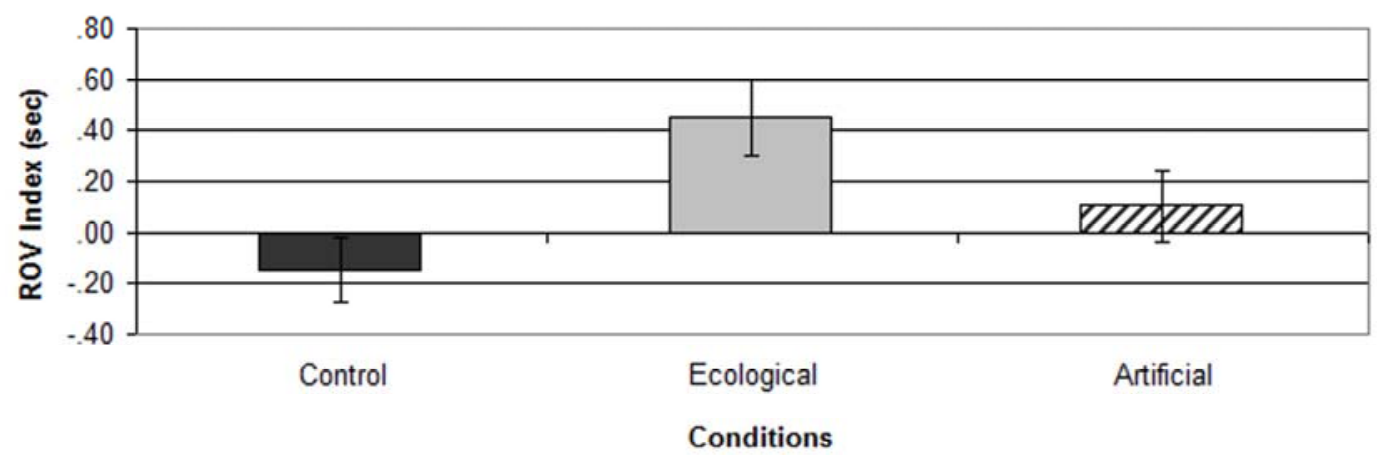

Figure 2. 